\title{
鮈亚科鱼类下咽骨和下咽齿的形态差异及其功能适应
}

\author{
曾 燏 ${ }^{1,2}$, 刘焕章 1 ,* \\ (1. 中国科学院水生生物研究所 水生生物多样性与保护重点实验室, 湖北 武汉 430072; 2. 中国科学院研究生院, 北京 100039) \\ 摘要: 采用双筒解剖镜观察和手工绘图的方法, 研究鮈亚科鱼类 39 个代表种下咽骨和下咽齿的形态特征, \\ 分析鮈亚科鱼类下咽骨和下咽齿的形态差异及其功能适应。结果显示, 鮈亚科鱼类的下咽骨可以分为粗壮型、中 \\ 间型和狭长型 3 种形态类型; 下咽齿可以分为圆雉型、白齿型、粗壮侧扁型、侧扁型和极侧扁型 5 种形态类型。 \\ 鮈亚科鱼类下咽骨和下咽齿间呈现出多种形态组合，并相互配合共同处理不同类型的食物。下咽骨和下咽齿的形 \\ 态差异为鮈亚科鱼类摄食不同类型的食物、充分利用不同的生境资源在摄食器官多样性方面提供了保证。
}

\section{Morphological Variation of Pharyngeal Bones and Teeth in the Subfamily Gobioninae (Cypriniformes: Cyprinidae) and Its Functional Adaptations}

\author{
ZENG Yu ${ }^{1,2}$, LIU Huan-zhang, ${ }^{1, *}$ \\ (1. The Key Lab of Aquatic Biodiversity and Conservation, Institute of Hydrobiology, the Chinese Academy of Sciences, Wuhan 430072, China; \\ 2. Graduate University of Chinese Academy of Sciences, Beijing 100039, China)
}

\begin{abstract}
By binocular microscope examination and hand-drawing, we observed the pharyngeal bones and teeth of 39 Gobininae species to explore their morphological variations and functional adaptation. The results showed that, in the Gobioninae, pharyngeal bones could be divided into three morphological types: wide, intermediate, and narrow; pharyngeal teeth into five morphological types: conical, molar, coarsely compressed, compressed and extremely compressed. Different types of pharyngeal bones and teeth cooperate together to deal with different types of food. Combination of the pharyngeal bones and teeth in the Gobioninae exhibit various types, and this variation occurring in trophic apparatus provides the diverse feeding organ adapting for the ecological resource utilization and the different types of dietary habits.
\end{abstract}

Key words: Gobioninae; Pharyngeal bone and teeth; Morphological variation; Functional adaptation

摄食器官 (口、齿、㗒) 的形态和功能相适应 是生物进化过程中的关键特征之一。在一些类群 中, 如蚚蜴、丽鱼和蛇等, 摄食器官的形态差异能 显著地提高物种的生态适应度, 甚至能促使这些类 群出现适应性辐射 (Forsman, 1991; Hulsey, 2006; Warheit et al, 1999)。鱼类拥有位置不同、形态各 异和功能多样的齿, 包括领齿、颚骨、犁骨齿、咽
齿等。鲤科鱼类的第五对鳃弓特化为下咽骨，其有 齿面上着生一至多行形态各异的下咽齿。这些特化 的下咽骨和下咽齿是鲤科鱼类分类的重要依据 (Chu，1935; Wu et al，1981; Chen，1986，1987; Zhou, 1989; Zhou, 1990; Howes, 1991; Miranda \& Escala, 2005), 同时, 它也是鲤科鱼类一个非常 重要的摄食器官（Eastman, 1970, 1971，1977;

收稿日期：2009-07-23; 接受日期：2009-10-15

基金项目：国家自然科学基金资助项目(40432003); 美国自然科学基金资助项目（CToL）

*通讯作者 (Corresponding author), Tel: 027-68780776, E-mail: hzliu@ihb.ac.cn

第一作者简介: 曾燏, 男, 博士研究生, 主要从事功能形态学研究 
Nakajima et al, 1981，1983; Sibbing, 1976，1982; Liem \& Sanderson, 1986; Vincent \& Sibbing, 1992)。 Sibbing (1982) 认为, 鲤科鱼类之所以能够发展成 为物种多样性最高的淡水鱼类类群之一, 其中一个 重要的原因就是拥有形态各异的下咽骨和下咽齿。 尽管一些学者对鲤科鱼类下咽骨、下咽齿的形态差 异及其系统分类应用, 下咽齿的发育, 咽骨、咽齿 的功能等进行了研究, 但时至今日, 有关鲤科鱼类 各类群下咽骨和下咽齿的形态差异及其功能适应 的研究鲜有报道。

鮈亚科 (Gobioninae) 是鲤科 (Cyprinidae) 中 物种比较多的亚科, 有近 30 个属和亚属, 共 190 余种及亚种，属中小型淡水鱼类，集中分布于东亚 水系，仅个别类群跨欧亚大陆分布 (Yue et al, 1998)。鮈亚科鱼类的形态多样, 其栖息的生态环 境和食性也存在极大的差异。同其他鲤科鱼类一 样, 鮈亚科也拥有发育良好的下咽骨和下咽齿, 其 下咽骨和下咽齿的形态呈现出巨大的差异, 是鮈亚 科鱼类演化过程中具有重要作用的器官。本研究以
鮈亚科鱼类代表物种的下咽骨和下咽齿为研究对 象, 观察和比较其形态差异，推测其不同的功能， 并讨论其与不同食性的适应性。

\section{1 材料和方法}

本文检测了鮈亚科 19 属共 39 种鱼类的下咽骨 和下咽齿的形态特征。所有标本均来自中国科学院 水生生物研究所标本馆 (表 1 )。

鮈亚科鱼类分类系统主要依据 Luo et al (1977)、Hosoya (1986)、Yue et al (1998) 和 Yang et al（2006）等关于鮈亚科鱼类的系统分类和系统 发育研究结果。本研究中鮈亚科鱼类的分类与鉴定 主要依据 Chen et al（1998）和 Yang et al（2006） 的系统。选择的物种代表了鮈亚科中 4 个不同亚类 群中在栖息生境、地理分布、食性及下咽骨和下咽 齿形态上具有差异的鱼类。

通过常规解剖方法, 将下咽骨和下咽齿一起从 福尔马林固定的全鱼样本中取出, 然后剔除下咽骨 和下咽齿上附着的黏液膜和肌肉, 用 $1 \%$ 的 $\mathrm{KOH}$ 溶

表 1 研究中所用的标本及来源

Tab. 1 Specimens and their localities in the present study

\begin{tabular}{|c|c|c|c|}
\hline 物种 Species & 拉丁名 Latin name & $\begin{array}{c}\text { 标本编号 } \\
\text { Specimen Nos. }\end{array}$ & 采集地 Locality \\
\hline 似刺鳊鮈 & Paracanthobrama guichenoti & 65XI067 & 江西九江 Jiujiang Jiangxi \\
\hline 似鳃 & Belligobio nummifer & 82VI2783 & 甘肃武都 Wudu Gansu \\
\hline 间鳃 & Hemibarbus medius & 75IV1091 & 广西崇左 Chongzuo Guangxi \\
\hline 大刺鱼骨 & Hemibarbus macracanthus & 83IV023 & 广西桂平 Guiping Guangxi \\
\hline 花刺鱼骨 & Hemibarbus umbrifer & 872083 & 贵州三都 Sandu Guizhou \\
\hline 长麦穗鱼 & Pseudorasbora elongata & 83IX2216 & 安徽石台 Shitai Anhui \\
\hline 细体鮈 & Gobio tenuicorpus & 89IX0248 & 吉林敦化 Dunhua Jilin \\
\hline 南方鮈 & Gobio meridionalis & 81VII1994 & 河南灵宝 Lingbao Henan \\
\hline 大头鮈 & Gobio macrocephalus & 645022 & 吉林晖春 Huichun Jilin \\
\hline 似鮈 & Pseudogobio vaillanti & $76 I V 1961$ & 广西翁源 Wengyuan Guangxi \\
\hline 桂林似鮈 & Pseudogobio guilinensis & $80 \times 1312$ & 广西阳朔 Yangshuo Guangxi \\
\hline 银鮈 & Squalidus argentatus & $80 \mathrm{VI} 1341$ & 陕西紫阳 Ziyang Shanxi \\
\hline 兴凯银鮈 & Squalidus chankaensis & 89IX0139 & 黑龙江牡丹江 Mudanjiang Heilongjiang \\
\hline 点纹银鮈 & Squalidus wolterstorffi & 63VI123 & 安徽金寨 Jinzhai Anhui \\
\hline 暗斑银鮈 & Squalidus atromaculatus & $76 I V 7459$ & 广东连平 Lianping Guangdong \\
\hline 湖南吻鮈 & Rhinogobio hunanensis & 8840437 & 湖南保靖 Baojing Hunan \\
\hline 圆筒吻鮈 & Rhinogobio cylindricus & 76VOII & 湖北丹江口 Danjiangkou Hubei \\
\hline 铜鱼 & Coreius heterodon & 63IV0312 & 江西湖口 Hukou Jiangxi \\
\hline 圆口铜鱼 & Coreius guichenoti & $83 \times 0413$ & 江西上饶 Shangrao Jiangxi \\
\hline
\end{tabular}


(接上表)

\begin{tabular}{|c|c|c|c|}
\hline 物种 Species & 拉丁名 Latin name & $\begin{array}{c}\text { 标本编号 } \\
\text { Specimen Nos. }\end{array}$ & 采集地 Locality \\
\hline 辽宁棒花鱼 & Abbottina liaoningensis & 8806038 & 辽宁太子河 Taizihe Liaoning \\
\hline 乐山小鳔鮈 & Microphysogobio kiatingensis & 79IV0302 & 四川乐山 Leshan Sichuan \\
\hline 福建小鳔鮈 & Microphysogobio fukiensis & 77V0017 & 浙江新安江 Xinanjiang Zhejiang \\
\hline 长体小鳔鮈 & Microphysogobio elongatus & 75IV2314 & 广西融安 Rongan Guangxi \\
\hline 洞庭小鳔鮈 & Microphysogobio tungtingensis & $82 \mathrm{~V} 2212$ & 贵州铜仁 Tongren Guizhou \\
\hline 光唇蛇鮈 & Saurogobio gymnocheilus & $90 \times 1021$ & 安徽洛溪口 Luoxikou Anhui \\
\hline 湘江蛇鮈 & Saurogobio xiangjiangensis & 9205805 & 江西梓树 Zishu Jiangxi \\
\hline 细尾蛇鮈 & Saurogobio gracilicaudatus & 76V006 & 湖北丹江口 Danjiangkou Hubei \\
\hline 嘉陵江领须鮈 & Gnathopogon herzensteini & 81VII14444 & 河南鲁山 Lushan Henan \\
\hline 短须领须鮈 & Gnathopogon imberbis & 45543 & 四川合川 Hechuan Sichuan \\
\hline 东北领须鮈 & Gnathopogon mantschuricus & 0579 & 黑龙江 Heilongjiang \\
\hline 济南领须鮈 & Gnathopogon tsinanensis & 82VI0132 & 陕西西周 Xizhou Shanxi \\
\hline 图们江中鮈 & Mesogobio tumenensis & 81IV0156 & 吉林和龙 Helong Jilin \\
\hline 片唇鮈 & Platysmacheilus exiguus & 87V056 & 贵州凯里 Kaili Guizhou \\
\hline 胡鮈 & Huigobio chenhsienensis & 90V2003 & 江西柔源 Wuyuan Jiangxi \\
\hline 福建华鳈 & Sarcocheilichthys sinensis fukiensis & 74VI2529 & 福建建瓯 Jianou Fujian \\
\hline 江西鳈 & Sarcocheilichthys kiangsiensis & 90VI877 & 江西柔源 Wuyuan Jiangxi \\
\hline 异鳔鳅鮀 & Xenophysogobio boulengeri & 78IV0215 & 四川乐山 Leshan Sichuan \\
\hline 南方鳅鮀 & Gobiobotia (Gobiobotia) meridionalis & 87V947 & 贵州凯里 Kaili Guizhou \\
\hline 宜昌鳅鮀 & Gobiobotia (Gobiobotia) filifer & 62V0091 & 四川乐山 Leshan Sichuan \\
\hline
\end{tabular}

液清洗, 茜素红染色, 最后保存在纯甘油中待进一 步观察。在双筒解剖镜下观察已制备好的下咽骨和 下咽齿的形态特征, 并手工绘制特征图。

下咽骨中所用形态术语参考 Chu (1935), 下咽 齿中所用形态术语参考 Nakajiam（1979，2008）, 下咽骨上所附着肌肉名称参考 Sibbing (1982)。

\section{2 结果}

\section{1 鮈亚科鱼类下咽骨的形态特点}

2.1.1下咽骨的形态及附着肌肉群的描述 鮈亚 科鱼类下咽骨可以分为两个面: 有孔面和有齿面。 有孔面上有数目、深浅不一的孔、洞或腔 (血管腔, blood vessel cavity) (图 1A); 有齿面与有孔面相对, 其上常着生有一行或多行下咽齿。

依据下咽骨在鱼体的位置可将其分为前端、后 端、腹侧和背侧 (图 1A)。下咽骨, 从前顶点到底 部中段的垂直距离为下咽骨的高 (L); 从有孔面腹 向最外侧到背向最内侧的最短距离为下咽骨的宽 (W)。下咽骨整个腹向区为下咽骨前臂, 从前臂
后端到整个向头背向弯曲区域为下咽骨的后臂。前 臂与后臂所形成的角为前角, 后臂背向区与头向区 所形成的角为后角。

下咽骨有齿面上附着有行使不同功能的肌肉, 主要有 7 块: 下弧共直肌（subarcualis rectus communis, SRC), 主要牵引下咽骨向前, 同时 也略向腹侧移动; 咽骨匙骨内前肌 (pharyngo-cleithralis internus anterior, PCIP), 主 要牟引下咽骨向后腹侧移动, 这样能扩大下咽骨与 前肠腔的容量; 咽骨匙骨外肌 (pharyngo-cleithralis externus, PCE), 主要牵引下咽骨腹侧和外侧运动; 第五腹膈肌 (transversus ventralis $\mathrm{V}, \mathrm{TV} \mathrm{V}$ ), 主要 使左右两边下咽骨向内侧收缩; 第五鳃弓提肌

(levator arcus branchialis V, LAB V), 主要向背部 提起下咽骨, 这样使下咽齿和碾磨垫相咬合; 咽骨 上缩肌 (retractor os pharyngeus superior, ROPS), 主要使下咽骨向后背部收缩, 同时协助主要缩肌 (咽骨下缩肌, ROPI) 的收缩运动, 从而促使咽齿 与碾磨垫相咬合; 咽骨下缩肌（retractor os 
pharyngeus inferior, ROPI), 主要使下咽骨向背后 向和中部收缩, 它是下咽骨位置的主要调节肌, 连 同 LAB V 和 ROPS 一起直接调控咽齿向后的咬合 运动 (Sibbing, 1982)。在鮈亚科鱼类中, PCIP 在 下咽骨有齿面上附着区域的形态差异极大。鮈亚鱼 类不同肌肉在下咽骨有齿面上的附着区见图 1B。

2.1.2 鮈亚科鱼类下咽骨的主要类型和功能 依 据下咽骨长与宽的比值范围以及 PCIP 附着区的形 态, 鮈亚科鱼类下咽骨有不同的形态类型和功能 (图 2)。
粗壮型 下咽骨宽短, 长与宽的比值范围为 1.82 3.16, PCIP 附着区弱或无。这种类型下咽骨 上附着的 ROPI 形态宽大, 而 PCIP 则比较窄小, 这 种类型下咽骨的咬合力量强大, 切割力量弱小, 代 表种类有铜鱼属和鳈属的鱼类。

中间型 下咽骨中等长短, 长与宽的比值范围 为 3.26 4.50, PCIP 附着区窄条状。这表示该类型 下咽骨上附着的 ROPI 和 PCIP 均为一般大小，这 种类型下咽骨具有一定的咬合力量, 也有一定的切

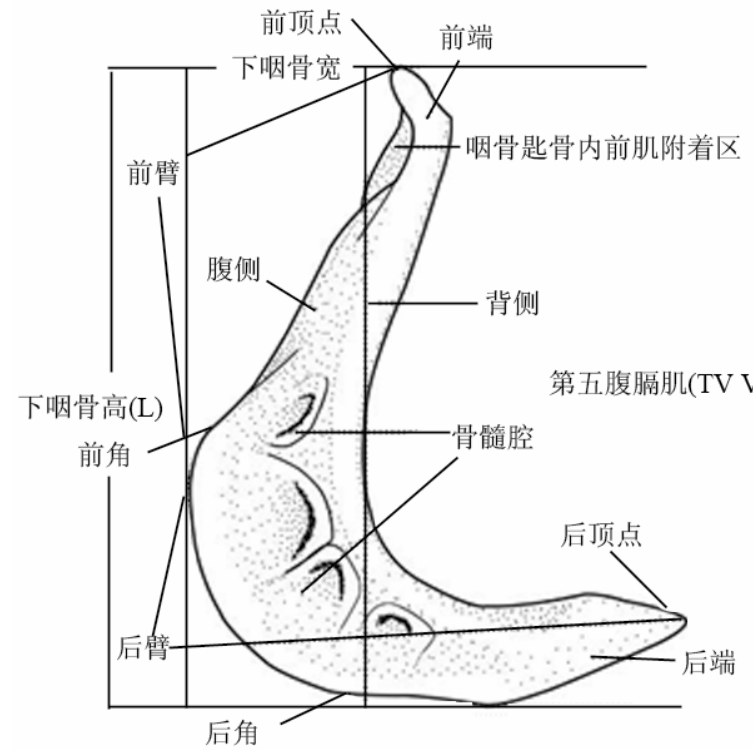

A

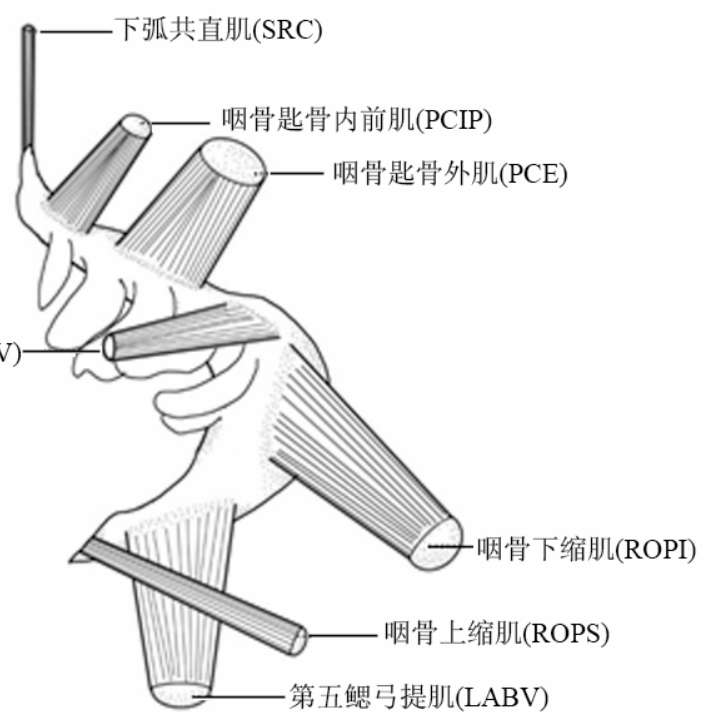

B

图 1 济南领须鮈下咽骨

Fig. 1 Pharyngeal bon of Gnathopogon tsinanensis

A: 下咽骨左侧有孔面; B: 下咽骨左侧有齿面及其上附着的 7 种肌肉。

A: Left side pharyngeal bone in pitted surface; B: Left side pharyngeal bone in dentulous surface.
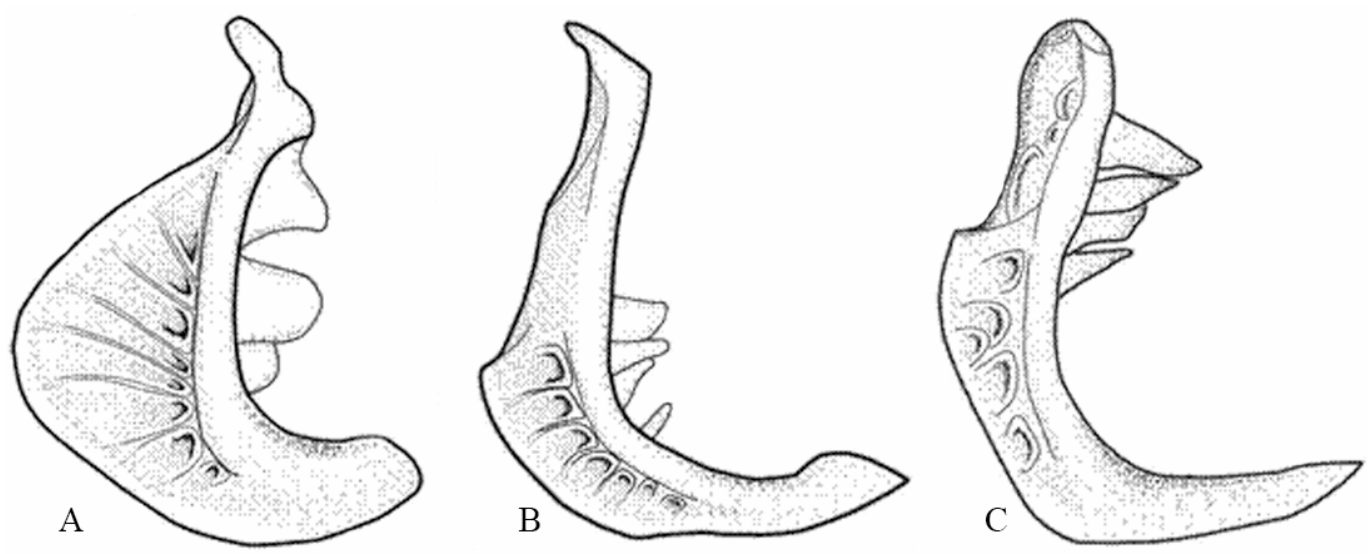

图 2 鮈亚科鱼类下咽骨的 3 种形态类型（依据 L/W 比值和 PCIP 附着区形态）

Fig. 2 Based on L/W and the morphology, the pharyngeal bone can be divided into three types

A: 粗壮型 (铜鱼); B: 中间型 (兴凯银鮈); C: 狭长型 (桂林似鮈)。

A: wide (Coreius heterodon); B: intermediate (Squalidus chankaensis); C: narrow (Pseudogobio guilinensis). 
割能力, 代表种类有银鮈属、鳃属和鮈属的一些种 类。

狭长型 下咽骨长而窄, 长与宽的比值范围为 4.51 7.47, PCIP 附着区片状向下腹侧延伸。这表 示该类型下咽骨上所附着的 ROPI 形态窄小, 而 PCIP 则宽大，这种类型下咽骨虽然咬合力量有限， 但切割力量强大, 代表种类有鳔鮈属、胡鮈属和蛇 鮈属的一些鱼类。

\section{2 鮈亚科鱼类下咽齿的形态结构和功能}

2.2.1 下咽齿的位置和功能 鮈亚科鱼类下咽骨 有齿面上着生有 $1 \sim 3$ 行下咽齿。参照 Nakajima
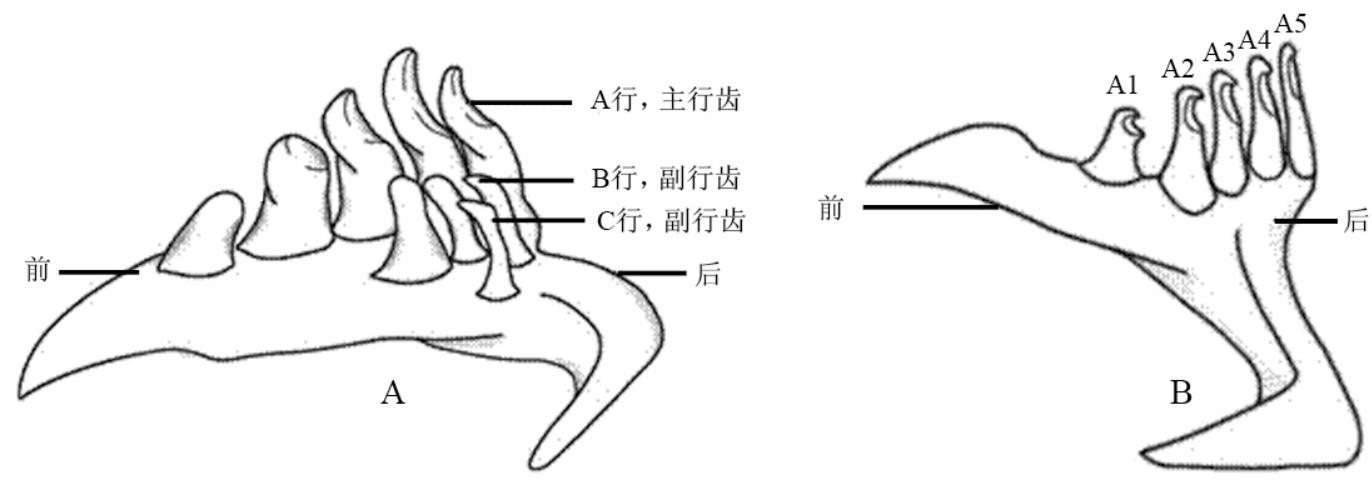

图 3 鮈亚科鱼类下咽齿主行齿和副行齿的排列（间鳃）(A）和主行齿的命名（洞庭小鳔鮈）(B)

Fig. 3 (A) the arrangement of the major row teeth and the minor row teeth (Hemibarbus medius); (B) the name of main row (Microphysogobio tungtingensis)

物进一步的加工处理，如碾磨或切碎等。

\subsection{2 下咽齿的形状和功能 鮈亚科鱼类主行齿} 形态多样, 主要有 5 种不同的形态类型 (图 4)。

A) 圆雉型齿, 齿圆锥形, 一般较小, 顶点尖, 直 立; B）臼型齿, 齿臼型, 较粗壮, 顶点针; C) 粗
（2008）的命名方法, 从最内侧起分别称为 A 行、 $B$ 行和 C 行。其中 A 行为主行齿, B 行和 C 行为副 行齿（图 $3 \mathrm{~A}$ )。在鮈亚科鱼类中，主行齿有 $4 \sim 5$ 枚不等，位于下咽骨有齿面的最内侧，是摄食功能 的主要执行者; 副行齿有 $1 \sim 2$ 行不等, 每行有 $1 \sim$ 4 枚不等，位于主行齿外侧，是主行齿执行摄食功 能的协助者。主行齿从前往后按顺序编号 (图 3 B), 如 A1 为主行齿最前面的第一枚齿，在摄食过程中 其最先接触到食物, 主要功能是对食物进行预处 理，如破除食物的坚硬外壳或进行初步切割等; $\mathrm{A} 2 \sim \mathrm{A} 5$ 为主行齿随后的几枚齿, 主要功能是对食
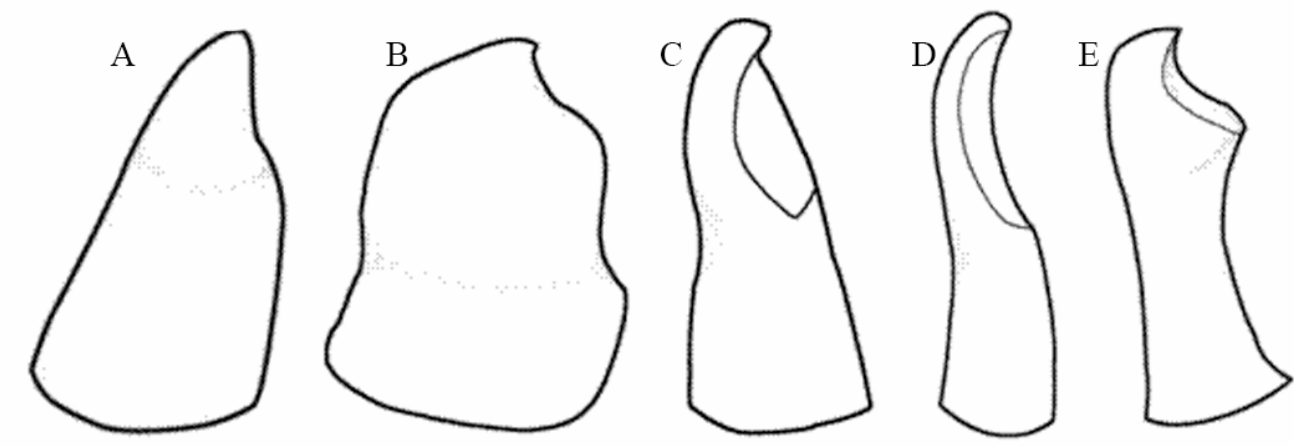

图 4 鮈亚科鱼类下咽齿的 5 种形态类型

Fig. 4 The five types of pharyngeal teeth

A：圆锥型； B：白齿型； C: 粗壮侧扁型； D: 侧扁型； E: 极扁侧扁型。

A: conical; B: molar; C: coarsely compressed; D: compressed; E: extremely compressed.

要功能是不一样的。对不同鱼类下咽齿的形态和食 性类型进行分析发现, 圆雉型齿主要出现在以鱼、
虾等为食的鱼类中, 因此其功能可能为刺破食物; 臼型齿主要出现在以蛙、蚬等软体动物为食的鱼类 
中, 其功能可能是碾磨食物; 极侧扁型齿则出现在 以水生植物为食的鱼类中, 其功能可能为切割食物

(Matthes, 1963; Sibbing, 1982; Eastman, 1997)。

因此, 在鮈亚科鱼类下咽齿中圆雉型齿、臼型 齿和极侧扁型齿的功能也应该分别为刺破食物表 皮结构, 碾磨食物坚硬的外壳和切割食物。另外, 粗壮侧扁型齿和侧扁型齿分别出现于鮈亚科中杂 食性偏动物食物和杂食性偏植物食性的类群中, 因 此推测粗壮侧扁型齿可能有相对较强的碾磨力量 和一定的切割力量, 而侧扁型齿则有相对较强的切 割力量和一定的碾磨力量。

2.2.3 下咽齿的组合和功能 鮈亚科鱼类下咽齿 行数也有很大差异, 有 1 3 行不等。下咽齿的行数 也是鲤科鱼类的一个重要分类性状 (Chu, 1935;

Cao et al, 1981; Hosoya, 1986)。依据下咽齿的行 数, 鮈亚科鱼类下咽齿有 3 种类型。

单行齿: 仅存 1 行主行齿, 代表种类有鳔鮈属、 蛇鮈属、似鮈属、铜鱼属和麦穗鱼属的鱼类;

两行齿: 主行齿 1 行, 副行齿 1 行, 代表种类 有鳅鮀属、银鮈属、鮈属、吻鮈属和领须鮈属的鱼 类。

多行齿: 主行齿 1 行, 副行齿 2 行, 且附行齿 都非常弱小，仅鱼骨属鱼类属于此类型。

在鮈亚科鱼类中, 一般情况下主行齿发达, 行 使主要功能。主行齿共有单一侧扁型、混合型和单 一臼齿型 3 种类型 (图 5)。

单一侧扁型主行齿: 所有主行齿均为极扁侧扁 型, 这种组合类型的主行齿能够提供较为强大的切 割力量, 代表种类有鳔鮈属和蛇鮈属的大多数种 类。

混合型主行齿: 主行齿由不同形态的下咽齿组
合而成, 有些种类为圆雉型和侧扁型相组合, 也有 白齿型和侧扁型相组合，该类型下咽齿组合能够同 时提供一定强度的咬合和切割力量。代表种类有鱼骨 属、银鮈属和鱾鮀属的种类。

单一臼齿型主行齿: 所有主行齿均为臼形齿, 该类型下咽齿的组合能够提供更为强大的咬合力 量, 代表种类有圆口铜鱼和蛇鮈。

\section{3 鮈亚科下咽骨和下咽齿的配合及差异}

虽然下咽骨和下咽齿有各自不同的形态特征 和功能, 但在摄食过程中下咽骨和下咽齿间是相互 协作的，不同形态的下咽骨和下咽齿间相互配合， 共同处理不同类型的食物（Vincent \& Sibbing, 1992)。依据下咽骨的类型及下咽齿的着生情况， 鮈亚科鱼类下咽骨和下咽齿有不同的组合形式及 功能。

粗壮型下咽骨有较强的咬合力量, 该类型的下 咽骨上可能着生臼齿型下咽齿齿, 共同加强咬合作 用, 如铜鱼和圆口铜鱼等。也有的粗壮下咽骨上着 生粗壮的侧扁型下咽齿, 行使咬合和切割的功能, 如似刺鳊鮈和福建华鳈等。

狭长型下咽骨常表示有较强的切割功能, 该类 型的下咽骨上可以着生极扁侧扁型下咽齿, 共同加 强切割作用, 如胡鮈和小鳔鮈等。也有狭长下咽骨 上着生兒齿和粗壮侧扁型下咽齿, 行使切割和咬合 的双重功能, 如图们江中鮈和异鳔鳅鮀等。

中等长短下咽骨则表明同时具备一定强度的 咬合和切割的力量，该类型的下咽骨上可以着生臼 齿型下咽齿, 加强其咬合方面的力量, 如湖南吻鮈 和银鮈等。也有中等长短的下咽骨上着生极扁侧扁 型下咽齿, 加强其切割方面的力量, 如桂林似鮈和 蛇鮈等, 这种类型的组合占鮈亚科鱼类的大多数。

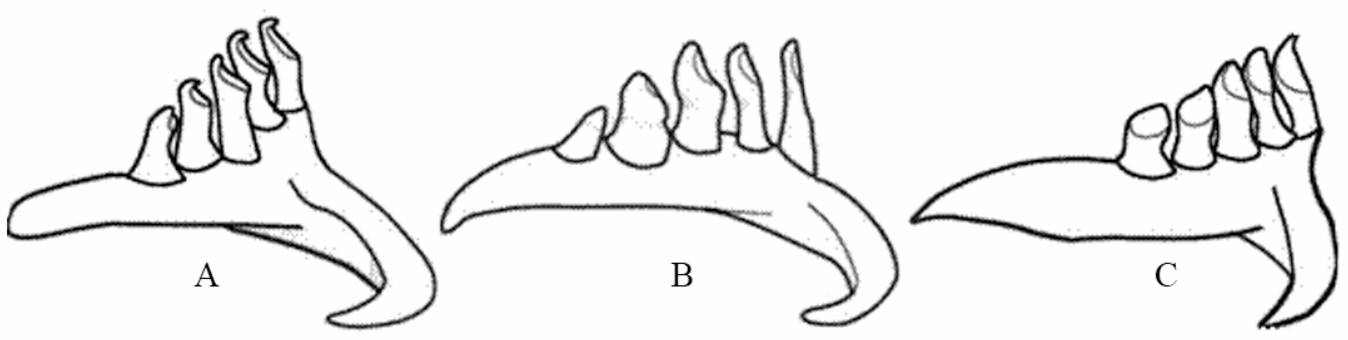

图 5 鮈亚科鱼类下咽齿 3 种不同的组合类型

Fig. 5 The three types combination of pharyngeal teeth

A: 单一侧扁型 (细尾蛇鮈); B: 混合型 (济南领须鮈); C: 单一臼齿型 (光唇蛇鮈)。

A: single compressed (Saurogobio gracilicaudatus); B: mixed (Gnathopogon tsinanensis); C: single molar (Saurogobio gymnocheilus). 


\section{3 讨 论}

\section{1 下咽骨和下咽齿在鮈亚科鱼类摄食过程中的 功能和作用}

鮈亚科鱼类有多种食性, 参照 Li (2001) 对食 性的划分方法, 鮈亚科鱼类可以分为以鱼虾和软体 动物为主的动物食性 (铜鱼和圆口铜鱼等), 以水 生昆虫和植物碎屑为主的杂食性 (领须鮈属和银鮈 属的鱼类) 和以腐屑、藻类和植物碎片等为主的植 物食性(似鮈、胡鮈和小鳔鮈等) (Yang \& Xie, 2005;

Ding et al，1994; FLHI, 1976)。

同其他鲤科鱼类一样, 作为重要摄食器官一部 分的下咽骨和下咽齿在鮈亚科鱼类咀嚼运动中的 过程也可以分为 3 个明显不同的阶段 (Sibbing 1982，1988; Vincent \& Sibbing, 1992; Lammens, 1985)。

（1）初始阶段 此阶段下咽骨、下咽齿和碾 磨垫相互远离, 这样可以使下咽齿和碾磨垫间的空 间增加到最大, 可以使食物进入恰当的咀嚼位置。

（2）执行阶段 此阶段下咽骨、下咽齿和碾 磨垫相互接近, 从而形成一个 “咽齿一食物一碾磨 垫的整合体 (tooth-food-chewing pad complex)”, 这 样能够对食物产生一个高强度的挤压力量。当齿面 和碾磨垫处于相互垂直状态时, 该整合体就能够发 挥高效的咬合力量; 当齿面和碾磨垫处于相互平行 状态时, 该整合体就能发挥高效的切割力量。

（3）结束阶段 此时 “咽齿一食物一碾磨垫 的整合体” 间的连接关系被打破。下咽骨、下咽齿 和碾磨垫处于相互远离, 此时下咽骨和下咽齿移向 一个调整的位置, 为下一个运动摄食过程做准备。

\section{2 鮈亚科鱼类下咽骨和下咽齿的形态差异及其 功能适应}

澄清动物摄食器官形态与功能的适应关系是 理解其形态和功能适应的关键 (Skulason \& Smith, 1995)。本研究显示, 鮈亚科下咽骨和下咽齿的形 态差异能高效的处理多种不同类型的食物。

首先, 对于下咽骨而言, 一般说来, 粗壮型下 咽骨上附着的 ROPI 形态宽大, 因而具备强大咬合 力量, 它适合处理具有坚硬外壳的食物; 中间型下 咽骨上附着的 ROPI 和 PCIP 均为一般大小, 能同 时具备一定强度的咬合和切割力量。它适合处理类 型复杂的食物; 狭长型下咽骨上 PCIP 附着区片状 向下腹侧延伸, 其形态宽大, 从而具备强大的切割 力量, 它适合处理较柔软或纤维含量高的食物。
其次, 对于不同形态类型的下咽齿, 圆锥型齿 和白形齿能够提供强大的穿刺和咬合的力量, 它能 高效地将食物的坚硬外壳刺破和咬碎; 粗壮侧扁型 下咽齿和侧扁型下咽齿, 它们也同时具备一定的咬 合和切割能力, 从而适合处理类型复杂的食物; 极 侧扁形下咽齿具有强大的切割力量, 它能高效地将 柔软或纤维成分高的食物切割成细小的片断。

我们的观察结果还表明, 鮈亚科下咽骨和下咽 齿存在多种不同的形态组合类型。下咽骨和下咽齿 的不同组合类型是与不同的生态功能和食性相适 应的。其中最基本的组合形式是中间型下咽骨上着 生粗壮侧扁型下咽齿, 这种组合类型同时具备一定 强度的咬合和切割力量, 它能适应复杂多变的生活 环境和食物类型, 为杂食性鱼类所拥有。在鮈亚科 中常见于一些较原始的类群 (Yue et al, 1998)。在 所观察的鮈亚科 19 个属中, 有 11 个属为该组合类 型, 包括麦穗鱼属、鰢鲫属、领须鮈属、吻鮈属、 鮈属、䲝鮀属、似鮈属、银鮈属、棒花鱼属、鰂属、 似鱼骨属和大多数蛇鮈属的鱼类。除基本组合类型 外, 下咽骨和下咽齿组合还表现出两种不同的特化 类型, 一种为粗壮型下咽骨上着生白形齿, 这种特 化类型具备强大的咬合力量, 它能适应摄食大型底 栖软体动物的生活习性, 为以幼蚌和螺蛳等为食的 动物食性鱼类所拥有, 常见于铜鱼属、鰁属和似刺 鳊鮈属的鱼类中; 另一种特化组合形式是狭长型下 咽骨上着生极扁侧扁型下咽齿, 这种组合类型具备 强大的切割力量, 它能适应摄食柔软或纤维含量较 高的食物, 为以水生昆虫、藻类和有机碎屑等为食 的植食性鱼类所拥有, 常见于中鮈属、异鳔鲜鮀属、 片唇鮈属、胡鮈属和小鳔鮈属的鱼类中。Ding （1994）和 FLHI（1976）对鮈亚科鱼类的食性有 较详细的描述, 本文划分的不同下咽骨、下咽齿类 型及其功能适应与这些食性的描述是相一致的。

另外, 本研究中还发现一个非常有趣的现象, 虽然鮈亚科下咽骨和下咽齿间存在多种理论上的 组合形式, 但在现实类群中有一些组合类型并未发 现, 如粗壮型下咽骨上就没有着生极扁侧扁形下咽 齿, 同样, 狭长型下咽骨上也没有着生臼形齿, 表 明下咽骨和下咽齿的组合并不是随机发生的。

总之, 鮈亚科鱼类下咽骨和下咽齿有多种形态 组合形式, 这为鮈亚科鱼类摄食不同类型的食物, 充分利用不同的生境资源在摄食器官多样性方面 提供保证。 


\section{参考文献:}

Cao WX, Chen YY, Wu YF, Zhu SQ. 1981. Origin and evolution of Schizothorscine fishes in relation to the upheaval of the Qinghai-Xizang Plateau.[M]// The comprehensive scientific expedition to the Qinghai-Xizang Plateau, CAS, ed. Studies on the Period, Amplitude and Type of the Uplift of the Qinghai-Xizang Plateau Beijng: Science Press, 118-130. [曹文宣, 陈宜瑜, 武云飞, 朱松泉. 1981. 裂腹鱼类的起源和演化及其与青藏高原隆起的关系 中国科 学院青藏高原综合科学考察队编.青藏高原隆起的时代、幅度和形 式问题. 北京: 科学出版社, 118-130.]

Chen XY. 1986. On the pharyngeal bones and teeth of Leuciscinae fishes (Cyprinidae)[J]. Zool Res, 7(2): 191-196. [陈星玉. 1986. 雅罗鱼亚科 咽骨及咽齿的研究. 动物学研究, 7(2): 191-196.]

Chen XY. 1987. Studies on the phylogenetic realationship of Chinese leuciscine fishes (Pisces: Cypriniformes)[J]. Acta Zootax Sin, 12(4): 427-438. [陈星玉. 1987. 中国雅罗鱼亚科的骨骼系统发育及其分类 学意义 (鲤形目: 鲤科). 动物分类学报. 12(4): 427-438.]

Chu YT. 1935. Comparative studies on the scales and on the pharyngeals and their teeth in Chinese cyprinids, with particular reference to taxonomy and evolution [D]. Biol Bull St John's Univ Shanghai, $1-225$.

Ding RH. 1994. Gobioninae. [A]. In: Ding RH. The Fishes of Sichuan[M]. Chengdu: Sichuan Publishing House of Science and Technology, 242-299. [丁瑞华. 1994. 鮈亚科. 见: 丁瑞华. 四川鱼类志. 成都: 四川科学技术出版社. 242-299]

Eastman JT. 1970. The pharyngeal bones and teeth of Minnesota cyprinid and catostomid fishes: functional morphology, variation and taxonomic significance [D]. Ph.D. thesis, University of Minnesota.

Eastman JT. 1971. The pharyngeal bone musculature of the carp, Cyprinus carpio[J]. J Morph, 134: 131-140.

Eastman JT. 1977. The pharyngeal bones and teeth of catostomid fishes[J]. Am Midl Nat, 97: 68-88.

Fish Laboratory of Hydrobiologica Institute in Hubei Province (FLHI). 1976. Fishes in the Yangtze River [M]. BeiJing: Sciences Press. [湖北 省水生生物研究所鱼类研究室. 1976. 长江鱼类. 北京: 科学出版 社.]

Forsman A. 1991. Adaptive variation in head size in Vipera berus L. populations [J]. Biol J Linn Soc, 43: 281-296.

Hosoya K. 1986. Interrelationships of the Gobioninae (Cyprinidae) [C]//Indo-Pacific Fish Biology: Proceeding of the Second International Conference on Indo-Pacific Fishes]. Tokyo: Ichthyological Society of Japan, 484-501.

Howes GJ. 1991. Systematics and Biogeography: An Overview [M]// Winfield, I. J. \& Nelson, J. S., eds. Cyprinid Fishes: Systematics, Biology and Exploitation. London: Chapman \& Hall,.1-33.

Hulsey CD. 2006. Function of a key morphological innovation: fusion of the cichlid pharyngeal jaw[J]. Proc R Soc Lond B, 273: 669-675.

Lammens EHRR. 1985. A test of a model for planktivorous filter feeding by bream Abramis brama[J]. Environ Biol Fish, 13: 288-296.

Li MD. 2001. Fish Ecology. 2nd ed. [M]. Tianjin: Department of Biology of Nankai University. [李明德. 2001. 鱼类生态学 (第二版). 天津: 南开大学生物系.]

Liem KF, Sanderson SL. 1986. The pharyngeal jaw apparatus of labrid fishes: A functional morphological perspective[J]. J Morph, 187: 143-158.

Luo YL, Yue PQ, Chen YY. 1977. Gobioninae. [A]. In: Wu XW. The Crprinoid Fishes of China (II) [M]. Shanghai: People's Press, 439-537 [罗云林, 乐佩琦, 陈宜瑜. 1977. 见: 伍献文. 中国鲤科鱼类志 (下

\section{卷）.上海：上海人民出版社, 439-537.]}

Matthes M. 1963. A comparative study of the feeding mechanisms of some African Cyprinidae (Pisces, Cyprinidae) [J]. Büdr Bierkunde, 33: 3-35.

Miranda R, Escala MC. 2005. Morphometrical comparison of cleithra, opercular and pharyngeal bones of autochthonous Leuciscinae (Cyprinidae) of Spain [J]. Folia Zool, 54(1-2): 173-188.

Nakajima T, Nakajima M, Yamazaki T. 2008. Evidence for fish cultivation during the Yayoi Period in Western Japan [J]. Int J Osteoarchaeol, 11: 232-246.

Nakajima T, Sugito M, Nakahara M, Ozaki M. 1981. An analysis on the pattern of tooth replacement in the cyprinid fish, Rhodeus ocellatus ocellatus [J]. Jpn J Oral Biol, 23: 893-895.

Nakajima T, Yoshida H, Sone B, Hotta Y. 1983. Replacement pattern of the pharyngeal teeth in cyprinid fish, Tribolodon hakonensis [J]. Jpn J Oral Biol, 25: 801-803.

Nakajima T. 1979. The development and replacement pattern of the pharyngeal dentition in the Japanese cyprinid fish, Gnathopogon coerulescens [J]. Copeia, 22-28.

Sibbing FA. 1976. Pharyngeal mastication in Cyprinus carpio(L.)[J]. Rev Trav Inst Peches Marit, 40: 744-745.

Sibbing FA. 1982. Pharyngeal mastication and food transport in the carp (Cyprinus curpio L.): a cineradiographic and electromyographic study [J]. J Morph, 172: 223-258.

Sibbing FA. 1988. Specializations and limitations in the utilization of food resources by the carp, Cyprinus carpio: a study of oral food processing [J]. Environ Biol Fish, 22: 161-178.

Skulason S, Smith TB. 1995. Resource polymorphisms in vertebrates [J]. Trends Ecol Evol, 10: 366-370.

Vincent JFV, Sibbing FA. 1992. How the grass carp (Ctenopharyngodon idella) chooses and chews its food: some clues [J]. J Zool London, 226: 435-444.

Warheit KI, Forman JD, Losos JB, Miles DB. 1999. Morphological diversification and adaptive radiation: a comparison of two diverse lizard clades [J]. Evolution, 53: 1226-1234.

Wu XW et al. 1977. The Crprinoid Fishes of China (II) [M]. Shanghai: People's Press. [伍献文等. 1977. 中国鲤科鱼类志 (下卷). 上海: 上 海人民出版社.]

Wu XW, Chen YY, Chen XL, Chen JX. 1981. Families division of the Cyprinidei and their systematic relationships[J]. Sci Sin, 3: 369-376. [伍献文, 陈宜瑜, 陈湘粦, 陈景星. 1981. 鲤亚目鱼类分科的系统 和科问系统发育的相互关系. 中国科学, 3: 369-376.]

Yang JQ, He SP, Freyhof J, Witte K, Liu HZ. 2006. The Phylogenetic mitochondrial cytochrome $b$ gene sequences[J]. Hydrobiologia, 553: 255-266.

Yang SX, Xie XM. 2005. Biological Characteristics of Coreius heterodon (Bleeker) and Its Potential as an Aquaculture Species[J]. Reserv Fish, 25(4): 33-34. [杨四秀, 谢新民. 2005. 铜鱼的生物学特性及养殖前 景分析. 水利渔业, 25(4): 33-34.]

Yue PQ. 1998. Gobioninae. [A]. In: Chen YY. Fauna Sinica. Osteichthyes. Cypriniformes II [M]. Beijing: Sciences Press, 232-378. [乐佩琦. 1998. 鮈亚科. 见: 陈宜瑜. 中国动物志 ・硬骨鱼纲 -鲤形目 (中卷). 北京: 科学出版社, 232-378]

Zhou JJ. 1990. The cyprinidae fossils from middle miocene of shanwang basin[J]. Vert PalAsiat, 28(2): 95-127. [周家健. 1990. 山东山旺中中 新世鲤科化石. 古脊椎动物学报, 28(2): 95-127.]

Zhou W. 1989. Phylogeny of the subfamily cyprininae (Pisces: Cyprinidae)[J]. Acta Zootax Sin, 14(2): 247-256. 